\title{
Prospective evaluation of the effect of adherent perinephric fat on outcomes of robotic assisted partial nephrectomy following elimination of the learning curve
}

\author{
Ashley M. Shumate ${ }^{1}$, Grayson Roth ${ }^{2}$, Colleen T. Ball ${ }^{3}$, David D. Thiel ${ }^{3}$ \\ ${ }^{1}$ Department of Urology, Mayo Clinic, Jacksonville, FL, USA; ${ }^{2}$ Department of Health Sciences Research, Mayo \\ Clinic, Jacksonville, FL, USA; ${ }^{3}$ Division of Biomedical Statistics and Informatics, Mayo Clinic, FL, USA
}

\section{ABSTRACT}

Purpose: To prospectively evaluate the association of adherent perinephric fat (APF) on perioperative outcomes of robotic-assisted partial nephrectomy (RAPN) following elimination of the surgical learning curve.

Materials and Methods: 305 consecutive RAPNs performed by a single experienced surgeon were analyzed. The first 100 RAPNs were considered the learning curve and therefore excluded. APF was defined as the necessity of subcapsular renal dissection to mobilize the tumor from surrounding perinephric fat. Perioperative outcomes were evaluated including operative time, warm ischemia time (WIT), postoperative complications, length of stay, margins, ischemia, and complications score (MIC), estimated blood loss (EBL), and change in pre-operative to postoperative day 1 (POD 1) laboratory values. After correction for multiple comparisons, $P$ values $\leq 0.0045$ were considered statistically significant but associations with $P$ values $\leq 0.05$ were also mentioned in the study results.

Results: Fifty-eight (28.3\%) patients had APF. Patients with APF had longer operative times compared to those without APF (median, 213 vs. 192 minutes, $\mathrm{P}<0.001$ ). There was some evidence of higher increase in change in creatinine from preoperative to POD 1 among those with APF compared to those without APF, although this was not statistically significant (median, $0.2 \mathrm{vs}$. $0.1 \mathrm{mg} / \mathrm{dL}, \mathrm{P}=0.03$ ). There were no other statistically significant associations between presence of APF and perioperative outcomes.

Conclusions: APF is associated with increased operative time but no change in other perioperative outcomes. Surgeon experience does not affect perioperative outcomes associated with APF.

\section{ARTICLE INFO}

Ashley M. Shumate

http://orcid.org/0000-0003-3035-9340

Keywords:

Carcinoma, Renal Cell;

Robotic Surgical Procedures;

Nephrectomy

Int Braz J Urol. 2019; 45: 1136-43

Submitted for publication:

February 10, 2019

Accepted after revision:

May 06, 2019

Published as Ahead of Print: August 01, 2019

\section{INTRODUCTION}

The learning curve of robotic assisted partial nephrectomy (RAPN) is steep. For surgeons already experienced with laparoscopic partial nephrectomy, the true learning curve has been proposed to be up to 65 cases, depending on the definition of proficiency (1). Adherent perinephric fat (APF), colloquially known as "sticky fat" can frustrate surgeons and complicate RAPN. Previous studies have shown APF to increase both operative time $(2,3)$ and estimated blood loss $(\mathrm{EBL}) \mathrm{du}-$ ring $\operatorname{RAPN}(4,5)$. There has also been an association of APF with increase in transfusion rate and 
conversion to either open or radical nephrectomy (6). However, to our knowledge, no study has examined the effect of surgeon experience and the surgical learning curve on the adverse outcomes associated with APF during RAPN. We previously demonstrated that APF prolonged operating room time of RAPN in a prospective evaluation of 100 patients (2). We examined if surgical experience eliminated the effect of APF on RAPN outcomes.

\section{MATERIALS AND METHODS}

\section{Data Collection}

All consenting patients undergoing surgery for a suspicious renal mass at our institution are prospectively included in an IRB-approved registry. We prospectively collect clinical, pathologic, and demographic data, as well as biological specimens (tumor tissue, blood, urine). At time of study, our registry included 305 patients who underwent RAPN by a single surgeon. To eliminate outcomes associated with the learning curve, the first 100 RAPN were excluded from our analysis. Adherent perinephric fat was recorded by the operative surgeon and defined as the necessity of subcapsular dissection to isolate the tumor from its surrounding perinephric fat (2).

We collected patient characteristics at baseline (age, sex, body mass index [BMI], preoperative creatinine, preoperative hemoglobin, preoperative estimated glomerular filtration rate [eGFR], hypertension, cardiovascular disease, diabetes, and history of smoking), tumor information (size of renal mass, R.E.N.A.L. nephrometry score (7), and Mayo Adhesive Probability [MAP] score (8)), operative information (EBL, operative time, warm ischemia time (WIT), conversion to open partial nephrectomy or laparoscopic nephrectomy, and surgical margins [positive or negative]) and postoperative information (hemoglobin at postoperative day [POD 1], creatinine at POD 1, eGFR at POD 1, postoperative complication grade $\mathrm{I}-\mathrm{V}$, and length of hospital stay [LOS]). Postoperative complications were catergorized by Clavien-Dindo grade (9). We additionally defined margins, ischemia, and complications (MIC) as those who had negative surgical margins, a warm ischemia time less than 20 minutes, and no postoperative complications higher than grade II (10). MAP and R.E.N.A.L. scores were calculated according to the algorithms previously described $(7,8)$. MAP scores of $0-3$ were considered low and scores of 4-5 considered high. R.E.N.A.L. scores were grouped 4-6, 7-9, and $10-12$, respectively. For patients with multiple renal masses, the size of the largest resected mass was used for analysis. All RAPN were performed in standard fashion as previously described utilizing sliding-clip renorrhaphy $(2,11)$.

\section{Statistical analysis}

Associations of perioperative outcomes with presence of APF and high MAP score $(>3)$ were evaluated using the Wilcoxon rank-sum test for continuous outcomes and the Fisher's exact test for categorical outcomes. Associations of perioperative outcomes with BMI and R.E.N.A.L. score were evaluated using the Spearman rank correlation test for continuous outcomes and the Cochran-Armitage trend test for categorical outcomes. To account for the increase in the likelihood of a type I error (i.e., a false-positive finding) secondary to the number of statistical tests performed, we used a Bonferroni correction separately when evaluating associations of perioperative outcomes with APF, BMI, R.E.N.A.L. score, and MAP score. After correction for multiple comparisons, $P$ values $\leq 0.0045$ were considered statistically significant but associations with $\mathrm{P}$ values $\leq 0.05$ were also mentioned in the study results. All analyses were performed using SAS (version 9.4, SAS Institute Inc., Cary, NC).

\section{RESULTS}

Among the 205 patients, 58 (28.3\%) had APF detected during RAPN. Patient and renal mass characteristics of the cohort are summarized in Table-1. Comparisons of perioperative outcomes between patients with APF and patients without APF are displayed in Table-2. Patients with APF had longer operative times compared to patients without APF (median, 213 vs. 192 minutes; $\mathrm{P}<0.001$ ). No other associations between presence of APF and perioperative outcomes were statistically significant after applying the Bonferroni 
Table 1 - Patient and renal mass characteristics.

\begin{tabular}{|c|c|}
\hline Characteristic & All patients $(n=205)$ \\
\hline Age, years & $63(22,56,71,84)$ \\
\hline \multicolumn{2}{|l|}{ Age distribution, n (\%) } \\
\hline$<60$ years & $76(37.1 \%)$ \\
\hline $60-65$ years & $34(16.6 \%)$ \\
\hline$>65$ years & $95(46.3 \%)$ \\
\hline Female sex, $\mathrm{n}(\%)$ & $82(40.0 \%)$ \\
\hline Body mass index, $\mathrm{kg} / \mathrm{m}^{2}$ & $28.7(16.5,25.6,33.0,60.6)$ \\
\hline \multicolumn{2}{|l|}{ Body mass index distribution, $n$ (\%) } \\
\hline$<25 \mathrm{~kg} / \mathrm{m}^{2}$ & $43(21.0 \%)$ \\
\hline $25-30 \mathrm{~kg} / \mathrm{m}^{2}$ & $78(38.0 \%)$ \\
\hline$>30 \mathrm{~kg} / \mathrm{m}^{2}$ & $84(41.0 \%)$ \\
\hline Preoperative creatinine, $\mathrm{mg} / \mathrm{dL}$ & $1.0(0.5,0.8,1.1,3.1)$ \\
\hline Preoperative hemoglobin & $13.9(10.7,12.8,14.7,18.0)$ \\
\hline Preoperative eGFR $<60 \mathrm{~mL} / \mathrm{min}, \mathrm{n}(\%)$ & $32(15.6 \%)$ \\
\hline Hypertension, n (\%) & $131(63.9 \%)$ \\
\hline Cardiovascular disease, $\mathrm{n}(\%)$ & $36(17.6 \%)$ \\
\hline Diabetes, $\mathrm{n}(\%)$ & $41(20.0 \%)$ \\
\hline History of smoking, $n(\%)$ & $59(28.8 \%)$ \\
\hline Size of renal mass, $\mathrm{cm}$ & $3.1(1.0,2.5,4.2,10.0)$ \\
\hline Adherent perinephric fat, $\mathrm{n}(\%)$ & $58(28.3 \%)$ \\
\hline RENAL nephrometry score & $8(4,7,9,11)$ \\
\hline \multicolumn{2}{|l|}{ RENAL nephrometry score distribution, $n(\%)$} \\
\hline $4-6$ & $46(22.4 \%)$ \\
\hline $7-9$ & $109(53.2 \%)$ \\
\hline $10-12$ & $50(24.4 \%)$ \\
\hline Mayo Adhesive Probability score & $1(0,0,4,5)$ \\
\hline Mayo Adhesive Probability score > $3, \mathrm{n}(\%)$ & $59(28.9 \%)$ \\
\hline
\end{tabular}

Data are given as the median (minimum, $25^{\text {th }}$ percentile, $75^{\text {th }}$ percentile, maximum) or number (percent). Mayo adhesive probability score was not available for 1 patient. 
Table 2 - Comparisons of perioperative outcomes according to presence of adherent perinephric fat.

\begin{tabular}{|c|c|c|c|}
\hline Perioperative outcome & Adherent Perinephric Fat $(n=58)$ & $\begin{array}{l}\text { No Adherent Perinephric Fat } \\
\qquad(\mathrm{n}=147)\end{array}$ & $P$ value \\
\hline Operative time, min & $213(148,188,228,336)$ & $192(106,169,216,320)$ & $<0.001$ \\
\hline Warm ischemia time, min & $19(0,14,24,35)$ & $19(0,14,23,42)$ & 0.33 \\
\hline $\begin{array}{l}\text { Conversion to open or laparoscopic } \\
\text { nephrectomy, } \mathrm{n}(\%)\end{array}$ & $0(0.0 \%)$ & $5(3.4 \%)$ & 0.32 \\
\hline Estimated blood loss (mL) & $400(300,300,500,1200)$ & $300(100,300,500,1500)$ & 0.17 \\
\hline $\begin{array}{l}\text { Any postoperative complication, grade } \\
\mathrm{I-V}, \mathrm{n}(\%)\end{array}$ & $13(22.4 \%)$ & $31(21.1 \%)$ & 0.85 \\
\hline $\begin{array}{l}\text { Postoperative complication, grade III-V, } \\
\mathrm{n}(\%)\end{array}$ & $3(5.2 \%)$ & $11(7.5 \%)$ & 0.76 \\
\hline \multicolumn{4}{|l|}{$\begin{array}{l}\text { Change in laboratory measures } \\
\text { (preoperative to POD 1) }\end{array}$} \\
\hline Hemoglobin, mg/dL & $-2.3(-5.5,-3.2,-1.7,-0.2)$ & $-2.1(-11.2,-2.8,-1.6,0.0)$ & 0.30 \\
\hline Creatinine, $\mathrm{mg} / \mathrm{dL}$ & $0.2(-0.3,0.0,0.4,1.2)$ & $0.1(-0.3,0.0,0.3,1.1)$ & 0.034 \\
\hline eGFR $\leq 60, n(\%)$ & $24 / 47(51.1 \%)$ & $47 / 124(37.9 \%)$ & 0.16 \\
\hline Length of hospital stay, days & $2(1,2,3,25)$ & $3(1,2,3,25)$ & 0.56 \\
\hline Length of hospital stay > 3 days, $n(\%)$ & $14(24.1 \%)$ & $29(19.7 \%)$ & 0.57 \\
\hline MIC, n (\%) & $30(51.7 \%)$ & $79(55.2 \%)$ & 0.75 \\
\hline
\end{tabular}

POD = postoperative day; $\mathbf{e G F R}=$ estimated glomerular filtration rate; $\mathbf{M I C}=$ margins, ischemia, and complications.

Data are given as the median (minimum, $25^{\text {th }}$ percentile, $75^{\text {th }}$ percentile, maximum) or number (percent). The change in eGFR from preoperative to POD 1 was reported as the fraction (percent) of patients with an eGFR $\leq 60$ at POD 1 among only those who had a preoperative eGFR $>60$. P values result from the Wilcoxon rank-sum test or the Fisher's exact test. P values $\leq 0.0042$ were considered statistically significant after applying a Bonferroni adjustment. Warm ischemia time was not available for 3 patients whose surgery converted to nephrectomy. Laboratory measures at POD 1 were not available for 2 patients. MIC was not available for 4 patients.

adjustment ( $\mathrm{P} \leq 0.0045$ considered significant). However, there was some evidence of higher increase in change in creatinine from preoperative to POD 1 among those with APF compared to those without APF (median, 0.2 vs. $0.1 \mathrm{mg} /$ $\mathrm{dL}, \mathrm{P}=0.03$ ) but this was not reflected in number of patients with eGFR $\leq 60 \mathrm{~mL} / \mathrm{min} / 1.73 \mathrm{~m}^{2}$. Conversion to laparoscopic nephrectomy was required in 5 cases, none of which were in patients with APF.

Associations of BMI with perioperative outcomes are shown in Table-3. There were no statistically significant associations after ad- justment for multiple comparisons or even notable associations of BMI with perioperative outcomes.

Comparisons of perioperative outcomes between patients with a low MAP score (0-3) and patients with a high MAP score (4-5) are presented in Table-4. Patients with a high MAP score had longer operative times compared to patients with a low MAP score (median, 213 vs. 192 minutes; $\mathrm{P}=0.002$ ). No other associations between presence of APF and perioperative outcomes were statistically significant after adjustment for multiple comparisons and there were no other notable associations (all $\mathrm{P} \geq 0.058$ ). 
Table 3 - Perioperative outcomes according to body mass index.

\begin{tabular}{|c|c|c|c|c|}
\hline Perioperative outcome & $\mathrm{BMI}<25 \mathrm{~kg} / \mathrm{m}^{2}(\mathrm{n}=43)$ & $\begin{array}{c}\text { BMI } 25-30 \mathrm{~kg} / \mathrm{m}^{2} \\
(\mathrm{n}=78)\end{array}$ & $\mathrm{BMI}>30 \mathrm{~kg} / \mathrm{m}^{2}(\mathrm{n}=84)$ & $P$ value \\
\hline Operative time, $\min$ & $\begin{array}{c}191(125,159,210 \\
240)\end{array}$ & $\begin{array}{c}198(106,175,227 \\
279)\end{array}$ & $\begin{array}{c}196(116,175,224 \\
336)\end{array}$ & 0.10 \\
\hline Warm ischemia time, min & $18(0,13,22,29)$ & $19(0,14,24,42)$ & $19(0,14,24,34)$ & 0.55 \\
\hline $\begin{array}{l}\text { Conversion to open or laparoscopic } \\
\text { nephrectomy, } \mathrm{n}(\%)\end{array}$ & $1(2.3 \%)$ & $3(3.8 \%)$ & $1(1.2 \%)$ & 0.55 \\
\hline Estimate blood loss (mL) & $\begin{array}{l}300(200,300,400 \\
800)\end{array}$ & $\begin{array}{c}300(100,300,500 \\
1200)\end{array}$ & $\begin{array}{c}400(100,300,500 \\
1500)\end{array}$ & 0.10 \\
\hline $\begin{array}{l}\text { Any postoperative complication, grade } \\
\mathrm{I}-\mathrm{V}, \mathrm{n}(\%)\end{array}$ & $13(30.2 \%)$ & $16(20.5 \%)$ & $15(17.9 \%)$ & 0.13 \\
\hline $\begin{array}{l}\text { Postoperative complication, grade III-V, } \\
\mathrm{n}(\%)\end{array}$ & $4(9.3 \%)$ & $4(5.1 \%)$ & $6(7.1 \%)$ & 0.77 \\
\hline \multicolumn{5}{|l|}{$\begin{array}{l}\text { Change in laboratory measures } \\
\text { (preoperative to POD 1) }\end{array}$} \\
\hline Hemoglobin, mg/dL & $\begin{array}{c}-2.2(-5.8,-3.0,-1.7 \\
-0.2)\end{array}$ & $\begin{array}{c}-2.2(-11.2,-3.1,-1.6 \\
0.0)\end{array}$ & $\begin{array}{c}-2.1(-5.0,-2.7,-1.6 \\
-0.5)\end{array}$ & 0.62 \\
\hline Creatinine, $\mathrm{mg} / \mathrm{dL}$ & $0.1(-0.2,0.0,0.3,0.7)$ & $0.2(-0.3,0.0,0.4,1.2)$ & $0.2(-0.3,0.1,0.4,1.1)$ & 0.079 \\
\hline $\mathrm{eGFR} \leq 60, \mathrm{n}(\%)$ & $9 / 36(25.0 \%)$ & $30 / 65(46.2 \%)$ & $32 / 70(45.7 \%)$ & 0.070 \\
\hline Length of hospital stay, days & $3(1,2,3,25)$ & $2(1,2,3,25)$ & $3(1,2,4,9)$ & 0.16 \\
\hline Length of hospital stay > 3 days, $\mathrm{n}(\%)$ & $6(14.0 \%)$ & $15(19.2 \%)$ & $22(26.2 \%)$ & 0.095 \\
\hline MIC, n (\%) & $23(54.8 \%)$ & $41(54.7 \%)$ & $45(53.6 \%)$ & 0.89 \\
\hline
\end{tabular}

$\mathbf{B M I}=$ body mass index; $\mathbf{P O D}=$ postoperative day; $\mathbf{E G F R}=$ estimated glomerular filtration rate; $\mathbf{M I C}=$ margins, ischemia, and complications.

Data are given as the median (minimum, $25^{\text {th }}$ percentile, $75^{\text {th }}$ percentile, maximum) or number (percent). The change in eGFR from preoperative to POD 1 was reported as the fraction (percent) of patients with an $\mathrm{EGFR} \leq 60$ at POD 1 among only those who had a preoperative eGFR $>60$. P values result from the Spearman rank correlation test or the Cochran-Armitage trend test. P values $\leq 0.0042$ were considered statistically significant after applying a Bonferroni adjustment.

\section{DISCUSSION}

RAPN is the most common minimally invasive technique currently utilized for nephron sparing in patients with small renal masses (12). The ability to predict which patients are at increased risk of complications and identifying risk factors remains crucial. Additionally, as the prevalence of overweight and obese patients continues to increase, determining how this increased adiposity affects surgical outcomes has become an important interest amongst surgeons. APF is one patient factor associated with adverse perioperative outcomes that can be predicted pre-operatively by radiographic and clinical variables but a full understanding of its pathophysiology remains elusive (8).

We sought to evaluate if surgeon experience eliminated the adverse outcomes associated with the presence of APF. Our study found that even after elimination of the surgical learning curve (100 cases in this study), presence of APF remained associated with longer operative time, suggesting surgeon expertise does not provide the 
Table 4 - Perioperative Outcomes according to the Mayo Adhesive Probability Score.

\begin{tabular}{|c|c|c|c|}
\hline Perioperative outcome & MAP 0-3 $(n=145)$ & MAP $4-5(n=59)$ & $P$ value \\
\hline Operative time, min & $192(106,170,216,320)$ & $213(135,183,228,336)$ & 0.002 \\
\hline Warm ischemia time, min & $19(0,14,23,42)$ & $19(0,14,24,35)$ & 0.68 \\
\hline $\begin{array}{l}\text { Conversion to open/laparoscopic } \\
\text { nephrectomy, } \mathrm{n}(\%)\end{array}$ & $4(2.8 \%)$ & $1(1.7 \%)$ & 1.00 \\
\hline Estimated blood loss (mL) & $300(100,300,500,1500)$ & $400(100,300,500,1200)$ & 0.45 \\
\hline $\begin{array}{l}\text { Any postoperative complication, grade } \\
\text { I-V, } n(\%)\end{array}$ & $32(22.1 \%)$ & $12(20.3 \%)$ & 0.85 \\
\hline $\begin{array}{l}\text { Postoperative complication, grade } \\
\text { III-V, } n(\%)\end{array}$ & $13(9.0 \%)$ & $1(1.7 \%)$ & 0.072 \\
\hline \multicolumn{4}{|l|}{$\begin{array}{l}\text { Change in laboratory measures } \\
\text { (preoperative to POD 1) }\end{array}$} \\
\hline Hemoglobin, mg/dL & $-2.1(-11.2,-2.8,-1.6,-0.2)$ & $-2.2(-5.5,-3.1,-1.6,0.0)$ & 0.68 \\
\hline Creatinine, $\mathrm{mg} / \mathrm{dL}$ & $0.1(-0.3,0.0,0.3,1.2)$ & $0.2(-0.3,0.1,0.4,0.6)$ & 0.058 \\
\hline $\mathrm{eGFR} \leq 60, \mathrm{n}(\%)$ & $45 / 121(37.2 \%)$ & $26 / 49(53.1 \%)$ & 0.062 \\
\hline Length of hospital stay, days & $3(1,2,3,25)$ & $2(1,2,3,7)$ & 0.77 \\
\hline Length of hospital stay > 3 days, $n(\%)$ & $28(19.3 \%)$ & $14(23.7 \%)$ & 0.57 \\
\hline MIC, n (\%) & $76(53.5 \%)$ & $32(55.2 \%)$ & 0.88 \\
\hline
\end{tabular}

MAP = Mayo adhesive probability score; $\mathbf{P O D}=$ postoperative day; $\mathbf{E G F R}=$ estimated glomerular filtration rate; $\mathbf{M I C}$, margins, ischemia, and complications.

Mayo adhesive probability score was not available for 1 patient. Data are given as the median (minimum, $25^{\text {th }}$ percentile, $75^{\text {th }}$ percentile, maximum) or number (percent). The change in eGFR from preoperative to POD 1 was reported as the fraction (percent) of patients with an eGFR $\leq 60$ at POD 1 among only those who had a preoperative eGFR $>60$. $P$ values result from the Wilcoxon rank-sum test or the Fisher's exact test. $P$ values $\leq 0.0042$ were considered statistically significant after applying a Bonferroni adjustment.

ability to overcome the difficulties associated with tumor dissection and isolation presented by APF. The outcomes in this study are similar to those found in our initial evaluation of the association of RAPN on perioperative outcomes (2). The results of this analysis and our original analysis do not reflect other literature which has found an increase in EBL in patients with APF undergoing RAPN (13). As such, this information could play a vital role not only in patient counseling pre-operatively but also in setting patient and surgeon expectations. There may also be a role for placing APF as a modifier to signify increased surgical difficulty in patients undergoing RAPN if surgeon experience does not eliminate the variation in operative time associated with the procedure.
The incidence of APF in previous publications has been noted to be between 10.6 to 55.2\% (13) and the true incidence likely falls somewhere near the middle of these, with the finding of just over $28 \%$ in our patient population likely reflecting this. This finding is consistent from our initial analysis of APF (30\% in the initial study).

When analyzing the perioperative outcomes between patients with low $(\leq 3)$ or a high $(>3)$ MAP score, the same outcome of increased operative time was found in both the APF and high MAP score group. The MAP score is a validated scoring system that can be used to predict the presence of APF (4-6, $8,14)$. Interestingly, the median operative time was equivalent between patients with a low MAP score and no APF (192 minutes, each), and also those with a high MAP score and APF (213 minutes, each). This 
again supports the efficacy of the MAP score to predict APF and its utility for surgeons.

Although it can be a risk factor for APF, BMI in itself does not appear to be essential in determining operative difficulty, or at least, surgical outcomes, as supported by our lack of association between BMI and perioperative outcomes of RAPN.

Our study is not without limitations. APF remains a relatively subjective definition. Although some have attempted to define this more objectively $(5,15)$, a single objective definition of APF may be useful for standardization of future research for determining outcomes. Similarly, our study is based on the experience of one surgeon at a high volume institution for RAPN and may not be generalizable to all surgeons or facilities. Our prevalence of overweight and obese patients was high at $79 \%$. Although this is close to the national average in the United States $(71.2 \%$ of the population is overweight or obese), worldwide the rate is much lower (39\% of adults are overweight and 13\% are obese) (16, 17). Given that our study was conducted at a tertiary referral center in the United States, we believe this patient variable is indicative of a more complex cohort and may not be applicable worldwide. Our study sought to evaluate the effect of APF during RAPN after elimination of the learning curve. The learning curve for RAPN has been shown to be well below 100 cases for experienced surgeons. We chose the generous, though arguably arbitrary number of 100 RAPN, for our decided learning curve benchmark. As this study found no significant difference in perioperative outcomes from prior studies and no improvement from our own prior work examining outcomes associated with APF, we cannot say with complete certainty the learning curve of surgically managing APF was eliminated, though we feel most experienced surgeons would agree with our benchmark. In addition, we tried to account for all variables that could possibly be significant to outcomes, but there may be other unaccountable factors that could serve as confounders to our results.

\section{CONCLUSIONS}

Regardless of surgeon experience and proficiency in RAPN, the presence of APF remains associated with longer operative times during RAPN. However, APF does not have an adverse association with other perioperative outcomes after elimination of the surgical learning curve.

\section{ABBREVIATIONS}

RAPN = robotic-assisted partial nephrectomy

$\mathrm{APF}=$ adherent perinephric fat

$\mathrm{EBL}=$ estimated blood loss

$\mathrm{BMI}=$ body mass index

eGFR = estimated glomerular filtration rate

MAP = Mayo adhesive probability

WIT $=$ warm ischemia time

POD = post-operative day

LOS $=$ length of stay

MIC $=$ margins, ischemia, and complications

\section{CONFLICT OF INTEREST}

None declared.

\section{REFERENCES}

1. Roman A, Ahmed K, Challacombe B. Robotic partial nephrectomy - Evaluation of the impact of case mix on the procedural learning curve. Int J Surg. 2016;29:132-6.

2. Davidiuk AJ, Parker AS, Thomas CS, Heckman MG, Custer $\mathrm{K}$, Thiel DD. Prospective evaluation of the association of adherent perinephric fat with perioperative outcomes of robotic-assisted partial nephrectomy. Urology. 2015;85:836-42.

3. Bylund JR, Qiong H, Crispen PL, Venkatesh R, Strup SE. Association of clinical and radiographic features with perinephric "sticky" fat. J Endourol. 2013;27:370-3.

4. Kocher NJ, Kunchala S, Reynolds C, Lehman E, Nie S, Raman JD. Adherent perinephric fat at minimally invasive partial nephrectomy is associated with adverse perioperative outcomes and malignant renal histology. BJU Int. 2016;117:636-41.

5. Dariane C, Le Guilchet T, Hurel S, Audenet F, Beaugerie $A$, Badoual C, et al. Prospective assessment and histological analysis of adherent perinephric fat in partial nephrectomies. Urol Oncol. 2017;35:39.e9-39.e17. 
6. Khene ZE, Peyronnet B, Mathieu R, Fardoun T, Verhoest G, Bensalah K. Analysis of the impact of adherent perirenal fat on peri-operative outcomes of robotic partial nephrectomy. World J Urol. 2015;33:1801-6.

7. Kutikov A, Uzzo RG. The R.E.N.A.L. nephrometry score: a comprehensive standardized system for quantitating renal tumor size, location and depth. J Urol. 2009;182:844-53.

8. Davidiuk AJ, Parker AS, Thomas CS, Leibovich BC, Castle EP, Heckman MG, et al. Mayo adhesive probability score: an accurate image-based scoring system to predict adherent perinephric fat in partial nephrectomy. Eur Urol. 2014;66:1165-71.

9. Dindo D, Demartines N, Clavien PA. Classification of surgical complications: a new proposal with evaluation in a cohort of 6336 patients and results of a survey. Ann Surg. 2004;240:205-13.

10. Buffi N, Lista G, Larcher A, Lughezzani G, Ficarra V, Cestari $A$, et al. Margin, ischemia, and complications (MIC) score in partial nephrectomy: a new system for evaluating achievement of optimal outcomes in nephron-sparing surgery. Eur Urol. 2012;62:617-8.

11. Bhayani SB, Figenshau RS. The Washington University Renorrhaphy for robotic partial nephrectomy: a detailed description of the technique displayed at the 2008 World Robotic Urologic Symposium. J Robot Surg. 2008;2:139-40.

12. Ghani KR, Sukumar S, Sammon JD, Rogers CG, Trinh $Q D$, Menon M. Practice patterns and outcomes of open and minimally invasive partial nephrectomy since the introduction of robotic partial nephrectomy: results from the nationwide inpatient sample. J Urol. 2014;191:907-12.
13. Lee SM, Robertson I, Stonier T, Simson N, Amer T, Aboumarzouk OM. Contemporary outcomes and prediction of adherent perinephric fat at partial nephrectomy: a systematic review. Scand J Urol. 2017;51:429-34.

14. Martin L, Rouviere 0, Bezza R, Bailleux J, Abbas F, SchottPethelaz AM, et al. Mayo Adhesive Probability Score Is an Independent Computed Tomography Scan Predictor of Adherent Perinephric Fat in Open Partial Nephrectomy. Urology. 2017;103:124-8.

15. Zheng Y, Espiritu P, Hakky T, Jutras K, Spiess PE. Predicting ease of perinephric fat dissection at time of open partial nephrectomy using preoperative fat density characteristics. BJU Int. 2014;114:872-80.

16. Hales CM, Fryar CD, Carroll MD, Freedman DS, Ogden CL. Trends in Obesity and Severe Obesity Prevalence in US Youth and Adults by Sex and Age, 2007-2008 to 2015-2016. JAMA. 2018;319:1723-5.

17. World Health Organization. Obesity and Overweight, 2018. available at. <https://www.who.int/news-room/fact-sheets/ detail/obesity-and-overweight>. Accessed April 5, 2019.

Correspondence address: David D. Thiel, MD Mayo Clinic, Department of Urology 4500 San Pablo Road, Jacksonville, FL 32224, USA Fax: +1 904 953-2218 E-mail: Thiel.david@mayo.edu 